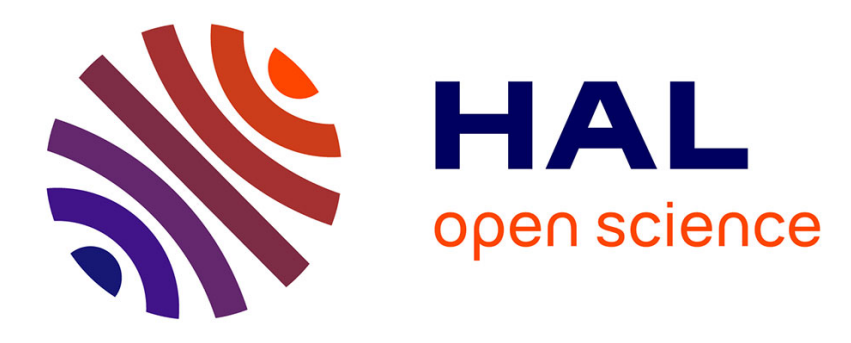

\title{
Influence of correlated collisions on Stark-broadened lines in plasmas
}

Joël Rosato, Hubert Capes, Roland Stamm

\section{To cite this version:}

Joël Rosato, Hubert Capes, Roland Stamm. Influence of correlated collisions on Stark-broadened lines in plasmas. Physical Review E: Statistical, Nonlinear, and Soft Matter Physics, 2012, Vol. 86, p.046407. hal-00945759

\section{HAL Id: hal-00945759 \\ https://hal.science/hal-00945759}

Submitted on 12 Feb 2014

HAL is a multi-disciplinary open access archive for the deposit and dissemination of scientific research documents, whether they are published or not. The documents may come from teaching and research institutions in France or abroad, or from public or private research centers.
L'archive ouverte pluridisciplinaire HAL, est destinée au dépôt et à la diffusion de documents scientifiques de niveau recherche, publiés ou non, émanant des établissements d'enseignement et de recherche français ou étrangers, des laboratoires publics ou privés. 


\title{
Influence of correlated collisions on Stark-broadened lines in plasmas
}

\author{
J. Rosato, H. Capes, and R. Stamm* \\ Aix-Marseille Université, CNRS, PIIM UMR 7345, \\ F-13397 Marseille Cedex 20, France
}

\begin{abstract}
An investigation of spectral line broadening in plasmas is carried out within a kinetic-theory approach, based on the Bogoliubov-Born-Green-Kirkwood-Yvon (BBGKY) hierarchy. The model employs a resummation procedure to account for correlated emitter-perturber collisions. Applications to hydrogen lines indicate that such collisions strongly affect the width and the shape in the core region. This argument is supported by comparisons to numerical simulations. It is also shown that the usual collision operator models, based on a binary description of emitter-perturber collisions, can be extremely inaccurate. The present model, in a better agreement with numerical simulations, is suggested as an extension suitable for the design of fast and accurate numerical routines for plasma diagnostics.
\end{abstract}

PACS numbers: $32.70 . \mathrm{Jz}, 32.60 .+\mathrm{i}, 52.25 . \mathrm{Dg}, 52.70 . \mathrm{Kz}$

*Electronic address: joel.rosato@univ-amu.fr 


\section{INTRODUCTION}

The Bogoliubov-Born-Green-Kirkwood-Yvon (BBGKY) hierarchy provides a valuable approach to the study of dilute medium such as gases or plasmas. The typical scheme for obtaining a kinetic equation consists in writing a closure relation based on suitable assumptions for the two-particle correlation function, and inserting this relation in the first equation of the hierarchy. This approach has been successfully used in the past for describing transport effects in plasmas (e.g., [1] for a review). In this work, we consider an adaptation of the BBGKY formalism to the description of Stark line shapes for plasma spectroscopy purposes and we focus on an extension devoted to retain correlated collisions between an emitter and the surrounding perturbers. Plasma spectroscopy is of general interest for diagnostics, given that the light naturally emitted from the atomic species (including multi-charged ions) contains information on the plasma parameters $\left(N, T_{e}\right.$, etc.) $[2-5]$. Detailed line shapes are also of interest for radiative transfer simulations, where they serve in transport codes as cross sections for the photon absorption and emission processes [6, 7]. It has been a long-standing challenge for plasma spectroscopy to accurately describe Stark-broadened line shapes [8]. The problem consists in a correct description of the atomic dipole autocorrelation function in the presence of the plasma microscopic electric field. The latter is created by numerous charged particles, so that one is confronted with a statistical time-dependent quantum problem which, even today, has no general analytical solution. A basic description of a line shape, sometimes referred to as the "standard model", consists in assuming the ions motionless during the dipole correlation time (or time "of interest") $\tau_{d d}$ and, at the same time, assuming the electrons moving rapidly. This short-time assumption for the electrons allows one to describe their contribution through a non-Hermitian part in the Hamiltonian, usually referred to as "collision operator". This quantity can be calculated using standard perturbation approaches, either semi-classical [9] or fully quantum-mechanical [10], using a binary atom-electron interaction model (impact approximation). The ionic contribution is described for its part with a constant Stark effect term in the Hamiltonian, and a statistical average over the ionic electric field is then performed using an appropriate probability density function. As a rule, deviations to the standard model are expected if the ordering $\tau_{e} \ll \tau_{d d} \ll \tau_{i}$ is not satisfied. Here, $\tau_{e}$ and $\tau_{i}$ denote the electron and ion collision times, estimated as $r_{0} / v_{e}, r_{0} / v_{i}$ with $v_{e}, v_{i}$ being the electron and ion thermal velocities, respec- 
tively, and $r_{0}=N^{-1 / 3}$ being the characteristic scale for the mean interparticle distance. Ion dynamics effects (that arise, for instance, if $\tau_{i} \sim \tau_{d d}$ ) have been observed [11] and have been extensively investigated, using numerical simulations [4, 12-15] or ad hoc models based on the statistical properties of the electric field (such as the model microfield method "MMM" $[16,17]$ or the frequency fluctuation model "FFM" [18-20]). Incomplete electron collisions (that are expected when $\tau_{e} \ll \tau_{d d}$ is not satisfied) have also been investigated, in particular by using refined models for the collision operator, either based on kinetic theory (such as the "unified theory" [21, 22]) or semiempirical procedures (e.g. using the Lewis cutoff [23]). Such models reproduce the result of the impact approximation at the line center (i.e., with the frequency detuning $\Delta \omega=0$ ) and yield an asymptotic behavior identical to that expected within the quasi-static approximation. Several works in the past have been devoted to generalizations of the collision operator models, able to account for non-impact effects, e.g. [24-26]. A major issue concerns the modeling of correlated collisions between the emitter and the charged particles surrounding it. An interpretation of the correlated collisions is that an emitter suffering a binary collision also "feels" the presence of the other particles. They are important when the characteristic collision frequency becomes of the same order as or larger than the inverse correlation time of the emitter-perturber interaction potential $[27,28]$. The latter is of the order of the inverse plasma frequency $\omega_{p}^{-1}$, and the collision frequency can be estimated as $N b_{W}^{2} v \ln \left(\lambda_{D} / b_{W}\right)$, where $N$ is the particle density, $v$ is the thermal velocity of the perturbers, $\lambda_{D}$ is the Debye length, and $b_{W}=\hbar n^{2} / m_{e} v$ is the socalled Weisskopf radius (here $n$ is the principal quantum number of the upper level). It determines an effective cross section which corresponds to collisions yielding coherence loss of the atomic wavefunction (strong collisions). The usual binary models assume that there are no simultaneous strong collisions, i.e. that the parameter $g=N b_{W}^{3}$ is small compared to unity. In general, correlated collisions concern both strong and weak collisions and, hence, may be important even for small $g$. In this work, we address the problem of modeling correlated collision effects on the line broadening with a first-principles approach inspired from the unified theory. The theoretical development makes extensive use of kinetic equations of BBGKY-type and follows early works reported on in the seventies [27, 28]. A preliminary attempt to retain correlated collisions has been performed by using a generalization of the Kirkwood truncature hypothesis to quantum operator [29]. Here, we follow a more rigorous approach based on a partial resummation of the correlation terms that arise in the BBGKY 
hierarchy. This procedure amounts to a renormalization of the atomic energy levels involved in the collision operator, accounting for their perturbation due to collisions. The method is designed for regimes where $g \lesssim 1$ and is suitable either for electron or ion collisions. We introduce the general formalism in Sec. II and, for the sake of simplicity, we specify it to one-component plasma in the weak coupling limit. This assumption allows one to consider the perturbers as non-interacting quasi-particles evolving on straight lines (ideal gas) and generating a Debye electric field. The resummation procedure is presented in Sec. III. We show that the collision operator is determined by a nonlinear equation and we examine an iterative scheme for solving it numerically. In order to examine the role of correlated collisions, we perform calculations in ideal cases and compare the results to the unified theory (Sec. IV). Comparisons with numerical simulations are also made. Finally, Sec. V reports on applications to realistic cases in the framework of magnetic fusion research.

\section{FORMALISM}

We give here a brief overview of the BBGKY approach to the line broadening problem, following the early works reported on in $[21,27,28]$. An atom immersed in a set of $\mathcal{N}$ charged particles is considered. For simplicity, we assume one-component plasma and we consider the particles evolving along straight lines and generating a Debye electric field. A generalization accounting for correlations between the perturbers in a rigorous way can be developed following [27]. A Stark line shape $I(\omega)$ is written as

$$
I(\omega)=\frac{1}{\pi} \operatorname{Re} \sum_{\varepsilon}\left\langle\left\langle\mathbf{d} \cdot \varepsilon\left|\tilde{\Phi}_{0}(-i \omega)\right| \rho \mathbf{d} \cdot \varepsilon\right\rangle\right\rangle
$$

Here, the double ket notation for Liouville space $|\ldots\rangle\rangle$ has been used, $\rho$ is the atomic density operator, $\mathbf{d} \cdot \boldsymbol{\varepsilon}$ is the dipole projected onto the polarization vector $\boldsymbol{\varepsilon}$, and $\tilde{\Phi}_{0}(-i \omega)=$ $\int_{0}^{+\infty} d t e^{i \omega t} \Phi_{0}(t)$ is the Laplace transform of the evolution operator averaged over the perturber trajectories (classical path assumption). The latter is obtained from the following definition with $p=0$

$$
\Phi_{s}(1 \ldots p ; t)=\int d(p+1) \ldots d \mathcal{N} f_{\mathcal{N}}(1 \ldots \mathcal{N}) U(1 \ldots \mathcal{N} ; t)
$$

Here, $1 \ldots \mathcal{N}$ stand for the phase space coordinates $\left(\mathbf{r}_{1}, \mathbf{v}_{1} \ldots \mathbf{r}_{\mathcal{N}}, \mathbf{v}_{\mathcal{N}}\right)$ of the perturbers, $d 1 \ldots d \mathcal{N}$ are the corresponding volume elements, $f_{\mathcal{N}}$ is the $\mathcal{N}$-particle phase space distribution, and 
$U$ is the atomic evolution operator. It obeys the Liouville equation

$$
\left\{\frac{\partial}{\partial t}+i L_{0}+\sum_{j=1}^{\mathcal{N}}\left[\mathbf{v}_{j} \cdot \frac{\partial}{\partial \mathbf{r}_{j}}+i V(j)\right]\right\} U=0,
$$

with the initial condition $U(t=0)=1$. All the quantities present in Eq. (3) are operators acting in the atomic Liouville space formed by the dyadics $|a b\rangle\rangle \equiv|a\rangle|b\rangle$. The term $L_{0}$ is the Liouvillian accounting for the atomic energy level structure and $V(j)=V\left(\mathbf{r}_{j}\right)=-\mathbf{d} \cdot \mathbf{E}\left(\mathbf{r}_{j}\right)$ denotes the Stark term resulting from the electric field due to the $j$-th perturber.

Equation (2) provides generalizations of the reduced phase space distributions, which account for the presence of the atom. It is customary to introduce a cluster expansion $(t$ is not written explicitly)

$$
\begin{cases}\Phi_{1}(1)= & f_{1}(1) \Phi_{0}+\Gamma_{1}(1) \\ \Phi_{2}(1,2)= & f_{2}(1,2) \Phi_{0}+f_{1}(1) \Gamma_{1}(2)+f_{1}(2) \Gamma_{1}(1)+\Gamma_{2}(1,2) \\ \Phi_{3}(1,2,3)= & f_{3}(1,2,3) \Phi_{0}+f_{2}(1,2) \Gamma_{1}(3)+f_{2}(1,3) \Gamma_{1}(2)+f_{2}(2,3) \Gamma_{1}(1) \\ & +f_{1}(1) \Gamma_{2}(2,3)+f_{1}(2) \Gamma_{2}(1,3)+f_{1}(3) \Gamma_{2}(1,2)+\Gamma_{3}(1,2,3) \\ \ldots & \end{cases}
$$

This decomposition allows one to write a hierarchy of equations for the correlations

$$
\begin{aligned}
& \left\{\frac{\partial}{\partial t}+i L_{0}+\sum_{j=1}^{p}\left[\mathbf{v}_{j} \cdot \frac{\partial}{\partial \mathbf{r}_{j}}+i V(j)\right]\right\} \Gamma_{p}(1 \ldots p) \\
& =-i \sum_{j=1}^{p} f_{1}(j) V(j) \Gamma_{p-1}(1 \ldots j-1, j+1 \ldots p)-i \mathcal{N} \int d(p+1) V(p+1) \Gamma_{p+1}(1 \ldots p+1),
\end{aligned}
$$

with the initial condition $\Gamma_{p}(1 \ldots p ; t=0)=0$ for $p \geq 1$. It has been assumed that the phase space distributions are space independent and factorize as $f_{p}(1 \ldots p)=f_{1}(1) \ldots f_{1}(p)$. Also, the thermodynamic limit $(\mathcal{N} \rightarrow \infty, \mathcal{V} \rightarrow \infty, \mathcal{N} / \mathcal{V}=$ cst with $\mathcal{V}$ being the volume of the system) is assumed. In Eq. (5), by convention $\Gamma_{0} \equiv \Phi_{0}$ and $\Gamma_{-1} \equiv 0$.

Originally, the unified theory was developed for applications in cases where the collisions are uncorrelated. The treatment involves equations for $\Phi_{0}$ and $\Gamma_{1}$ only, setting $\Gamma_{2} \equiv 0$ :

$$
\left(\frac{\partial}{\partial t}+i L_{0}\right) \Phi_{0}=-i \mathcal{N} \int d 1 V(1) \Gamma_{1}(1)
$$

and

$$
\left[\frac{\partial}{\partial t}+i L_{0}+\mathbf{v}_{1} \cdot \frac{\partial}{\partial \mathbf{r}_{1}}+i V(1)\right] \Gamma_{1}(1)=-i f_{1}(1) V(1) \Phi_{0}
$$


Equation (7) can be solved formally by using the propagator of the atom under the influence of one perturber, $Q(1, t)$. It obeys the following equation

$$
\left[\frac{\partial}{\partial t}+i L_{0}+i V\left(\mathbf{r}_{1}+\mathbf{v}_{1} t\right)\right] Q(1, t)=0,
$$

with the initial condition $Q(1, t=0)=1$, and it is proportional to a time-ordered exponential (Dyson series)

$$
Q(1, t)=e^{-i L_{0} t} \mathcal{T} \exp \left[-i \int_{0}^{t} d \tau e^{i L_{0} \tau} V\left(\mathbf{r}_{1}+\mathbf{v}_{1} \tau\right) e^{-i L_{0} \tau}\right]
$$

$\mathcal{T}$ being the time-ordering operator. The correlation $\Gamma_{1}$ is obtained from a convolution

$$
\Gamma_{1}(1 ; t)=-i f_{1}(1) \int_{0}^{t} d \tau Q\left(\mathbf{r}_{1}-\mathbf{v}_{1} \tau, \mathbf{v}_{1}, \tau\right) V\left(\mathbf{r}_{1}-\mathbf{v}_{1} \tau\right) \Phi_{0}(t-\tau)
$$

Inserting the solution Eq. (10) into the right-hand side of Eq. (6) provides a closed, integrodifferential equation for the averaged evolution operator $\Phi_{0}(t)$. Its solution takes a simple form in the Laplace space:

$$
\tilde{\Phi}_{0}(s)=\left[s+i L_{0}+K(s)\right]^{-1} .
$$

Inserting this relation in Eq. (1) provides an analytical expression for the line shape. Decomposing the double bra and kets onto an appropriate base of the Liouville space indicates that the line shape can be written as a sum of generalized Lorentzian functions, whose widths are frequency-dependent and given by matrix elements of $K(s=-i \omega)$. The latter quantity is a collision operator that accounts for incomplete collisions. It is completely determined in terms of the propagator $Q$ and the interaction term $V$ :

$$
K(s)=\mathcal{N} \int_{0}^{\infty} d t e^{-s t} \int d 1 V\left(\mathbf{r}_{1}+\mathbf{v}_{1} t\right) Q(1, t) V(1) f_{1}(1)
$$

\section{CORRELATED COLLISIONS}

The assumption $\Gamma_{2} \equiv 0$ considered in the unified theory is not valid in regimes where correlated collisions are present. Such correlations occur if, during the characteristic time for a binary collision (estimated as $\lambda_{D} / v \sim \omega_{p}^{-1}$ ), another perturber affects the atomic wavefunction. Such a perturbation occurs at a time scale of the order of the inverse matrix elements of the collision operator. A complete treatment of correlated collisions involving the infinite hierarchy Eq. (5) seems out of reach. To proceed further we propose a simplification 
that uses the singular role of the $p+1$-th particle in the $p+1$-correlation present in the integral term of Eq. (5). The approach is inspired from diagrammatic techniques used in kinetic theory ("ring approximation", e.g. [30]). For all $p \geq 1$, we assume that $p$-correlations are created or destroyed due to the $p$-th particle only. Therefore, the sums involving $V$ in Eq. (5) are replaced by their last term. The following evolution equation is obtained

$$
\begin{aligned}
& {\left[\frac{\partial}{\partial t}+i L_{0}+\sum_{j=1}^{p} \mathbf{v}_{j} \cdot \frac{\partial}{\partial \mathbf{r}_{j}}+i V(p)\right] \Gamma_{p}(1 \ldots p)} \\
& =-i f_{1}(p) V(p) \Gamma_{p-1}(1 \ldots p-1)-i \mathcal{N} \int d(p+1) V(p+1) \Gamma_{p+1}(1 \ldots p+1) .
\end{aligned}
$$

To solve this system $(p \geq 1)$ it is practical to use operator techniques in the Laplace space. We define the resolvent

$$
G_{p}(z)=\left[z-L_{0}+i \sum_{j=1}^{p} \mathbf{v}_{j} \cdot \frac{\partial}{\partial \mathbf{r}_{j}}-V(p)\right]^{-1},
$$

the inversion being taken in the operator sense, and we introduce two operators $A$ and $P$ acting on the correlations

$$
\begin{gathered}
\left(A \tilde{\Gamma}_{p-1}\right)(1 \ldots p)=G_{p}(i s) f_{1}(p) V(p) \tilde{\Gamma}_{p-1}(1 \ldots p-1) \\
\left(P \tilde{\Gamma}_{p+1}\right)(1 \ldots p)=G_{p}(i s) \mathcal{N} \int d(p+1) V(p+1) \tilde{\Gamma}_{p+1}(1 \ldots p+1) .
\end{gathered}
$$

These operators can be interpreted as creating and destroying correlations, respectively. The evolution equation (13) can be written in the Laplace space in a compact form

$$
\tilde{\Gamma}_{p}=A \tilde{\Gamma}_{p-1}+P \tilde{\Gamma}_{p+1}
$$

We solve this equation using the ansatz $P \tilde{\Gamma}_{p+1}=C \tilde{\Gamma}_{p}$ where $C$ depends on $A$ and $P$. This relation stems from the fact that $\lim _{p \rightarrow+\infty} \Gamma_{p}=0$ is assumed. A recurrence relation for $\tilde{\Gamma}_{p}$ is obtained

$$
\tilde{\Gamma}_{p}=(1-C)^{-1} A \tilde{\Gamma}_{p-1}
$$

This leads to a formal solution of Eq. (17):

$$
\tilde{\Gamma}_{p}=\left[(1-C)^{-1} A\right]^{p} \tilde{\Phi}_{0}
$$

Here we have again used the convention $\tilde{\Gamma}_{0} \equiv \tilde{\Phi}_{0}$. The latter quantity is obtained from Eq. (17) with $p=0, A \tilde{\Gamma}_{-1} \equiv i G_{0}(i s)$, using that $P \tilde{\Gamma}_{1}=C \tilde{\Phi}_{0}$

$$
\tilde{\Phi}_{0}=i(1-C)^{-1} G_{0}(i s) .
$$


A closed relation for $C$ is obtained from Eq. (18), multiplying on the left by $P$ and again using the ansatz $\left(P \tilde{\Gamma}_{p}=C \tilde{\Gamma}_{p-1}\right)$ :

$$
C=P(1-C)^{-1} A
$$

This equation is transcendental because $P$ is not invertible. A resolution should therefore involve an iterative scheme.

The collision operator is obtained from Eq. (20), performing algebraic manipulations and identifying with the terms in Eq. (11)

$$
K(s)=i G_{0}^{-1}(i s) C_{0}
$$

Here, $C_{0}$ denotes a restriction of $C$ such that $C \Phi_{0} \equiv C_{0} \Phi_{0}$. This quantity is determined using the recurrence relation $(p \geq 0)$

$$
C_{p}=P_{p}\left(1-C_{p+1}\right)^{-1} A_{p+1}
$$

which stems from Eq. (21) and where $A_{p}, P_{p}$ are restrictions of $A$ and $P$ such that $A \Gamma_{p} \equiv$ $A_{p+1} \Gamma_{p}$ and $P \Gamma_{p} \equiv P_{p-1} \Gamma_{p}$. We define a set of operators $K_{0}, K_{1}, K_{2} \ldots$ generalizing the collision operator, in such a way that the following property is satisfied

$$
K_{p}(1 \ldots p ; s)=i G_{p}^{-1}(i s) C_{p}
$$

These operators obey the following recurrence relation (the dependence on $1 \ldots p, p+1$ is not written explicitly)

$$
K_{p}(s)=i G_{p}^{-1}(i s) P_{p}\left[1+i G_{p+1}(i s) K_{p+1}(s)\right]^{-1} A_{p+1},
$$

or, using the explicit definition of $A_{p+1}$ and $P_{p}$ and making elementary algebra

$$
K_{p}(s)=i \mathcal{N} \int d(p+1) V(p+1)\left[G_{p+1}^{-1}(i s)+i K_{p+1}(s)\right]^{-1} V(p+1) f_{1}(p+1) .
$$

We simplify this relation by using the ansatz $K_{p}(1 \ldots p ; s)=K\left(s+\sum_{j=1}^{p} \mathbf{v}_{j} \cdot \partial / \partial \mathbf{r}_{j}\right)$, which stems from the presence of $i s+i \sum_{j=1}^{p} \mathbf{v}_{j} \cdot \partial / \partial \mathbf{r}_{j}$ in the denominator. Setting $p \equiv 0$ in Eq. (26) yields a closed equation for the collision operator

$$
K(s)=i \mathcal{N} \int d 1 V(1) \bar{G}_{1}(i s) V(1) f_{1}(1)
$$


where $\bar{G}_{1}$ is a modified resolvent

$$
\bar{G}_{1}(z)=\left[z-L_{0}+i \mathbf{v}_{1} \cdot \frac{\partial}{\partial \mathbf{r}_{1}}-V(1)+i K\left(-i z+\mathbf{v}_{1} \cdot \frac{\partial}{\partial \mathbf{r}_{1}}\right)\right]^{-1} .
$$

The latter can be interpreted as a propagator involving energy levels "dressed" by the presence of the other particles, through the presence of the collision operator as a non-Hermitian part in the Liouvillian $L_{0}$. In the case where the correlated collisions are neglected, $\bar{G}_{1} \equiv G_{1}$ and the collision operator reduces to that obtained within the binary approximation, Eq. (12). The set of equations (27) and (28) is nonlinear. It is practical to define an effective propagator $Q_{\text {eff }}(1, t)$, satisfying the dressed one-particle Schrödinger equation

$$
\left[\frac{\partial}{\partial t}+i L_{0}+i V\left(\mathbf{r}_{1}+\mathbf{v}_{1} t\right)\right] Q_{\mathrm{eff}}(1, t)+\int_{0}^{t} d \tau M(\tau) Q_{\mathrm{eff}}(1, t-\tau)=0,
$$

with the initial condition $Q_{\text {eff }}(1, t=0)=1$. Here $M(t)$ is the inverse Laplace transform of the collision operator. Equation (29) is interpretable as describing the evolution of the atom under the influence of one collision represented by the interaction term $V$, given a set of collisions occurring in its past history. These collisions act accumulatively and are taken into account by the kernel $M(t)$. The absence of this term in the unified theory [Eq. (8)] stems from the assumption $\Gamma_{2} \equiv 0$. The latter is valid provided the characteristic evolution time for $Q_{\text {eff }}$ be much shorter than the time between two collisions. In the case of weakly correlated plasma, the evolution time is of the order of $\lambda_{D} / v$, so that a validity criterion is provided by the relation $\lambda_{D} \gamma / v \ll 1$ where $\gamma$ is a typical matrix element of the collision operator, e.g. estimated as $\gamma=N b_{W}^{2} v \ln \left(\lambda_{D} / b_{W}\right)$. This result is in agreement with the analysis reported on in $[27,28]$ and it indicates that the present extension of the unified theory allows one to explore regimes where correlated collisions are present. In terms of the adimensional parameter $h=N b_{W}^{2} \lambda_{D} \ln \left(\lambda_{D} / b_{W}\right)$, such regimes correspond to $h \gtrsim 1$. The collision operator accounting for correlated collisions can be written in a fashion similar to that obtained within the unified theory:

$$
K(s)=\mathcal{N} \int_{0}^{\infty} d t e^{-s t} \int d 1 V\left(\mathbf{r}_{1}+\mathbf{v}_{1} t\right) Q_{\mathrm{eff}}(1, t) V(1) f_{1}(1) .
$$

The substitution of $Q$ by $Q_{\text {eff }}$ denotes a renormalization of the atomic energy levels induced by the correlated collisions. This stems from the structure of the modified resolvent Eq. (28). This is also illustrated by rewriting Eq. (29) in the Fourier and Laplace domain:

$$
\tilde{\hat{Q}}_{\mathrm{eff}}\left(\mathbf{k}_{1}, \mathbf{v}_{1}, s\right)=\left[s+i L_{0}+K(s)\right]^{-1}\left[(2 \pi)^{3} \delta\left(\mathbf{k}_{1}\right)-i \int \frac{d^{3} k_{2}}{(2 \pi)^{3}} \hat{V}\left(\mathbf{k}_{2}\right) \tilde{\hat{Q}}_{\mathrm{eff}}\left(\mathbf{k}_{1}-\mathbf{k}_{2}, \mathbf{v}_{1}, s-i \mathbf{k}_{2} \cdot \mathbf{v}_{1}\right)\right] \text {. }
$$


Here the convention $\hat{F}(\mathbf{k})=\int d^{3} r e^{-i \mathbf{k} \cdot \mathbf{r}} F(\mathbf{r})$ has been used for any function of space $F(\mathbf{r})$. As can be seen, the collision operator appears in the denominator as a non-Hermitian contribution to the Liouvillian. Equation (31) presents similarities with the result of the resonance broadening theory used for plasma turbulence [31, 32], where the quasi-linearized Vlasov equation plays a role similar to Eq. (29). In this theory, the coupling between the oneparticle distribution function and the plasma's electric field is described through a diffusion coefficient in the velocity space and the latter obeys a nonlinear equation as does our collision operator. Resonance broadening models have also been used in solid state physics for the calculation of dielectric functions (e.g., [33]).

In practice, a calculation of the collision operator from Eq. (30) should be done by iterations. A simplification, practical for numerical applications, is provided by assuming $K(s) \simeq K\left(-i \omega_{0}\right) \equiv K_{0}$ (with $\omega_{0}$ being the central frequency of the line under consideration)

in Eq. (31), using that the collision operator is governed by the values of $\tilde{\hat{Q}}_{\text {eff }}$ near the resonance. This amounts to setting $M(t) \equiv K_{0} \delta(t)$ in Eq. (29), and it leads to a simple expression for $Q_{\text {eff }}$, with a structure similar to that in the binary case Eq. (9):

$$
Q_{\text {eff }}(1, t)=e^{\left(-i L_{0}-K_{0}\right) t} \mathcal{T} \exp \left[-i \int_{0}^{t} d \tau e^{i L_{0} \tau} V\left(\mathbf{r}_{1}+\mathbf{v}_{1} \tau\right) e^{-i L_{0} \tau}\right]
$$

A simple expression of the collision operator for hydrogen Lyman lines, suitable for small $r_{0} / \lambda_{D}$, is derived in Appendix A. Figure 1 shows the convergence of the iterations in a specific case. The diagonal element of the collision operator for $\mathrm{Ly}-\alpha$ is plotted in terms of the number of iterations for $r_{0} / \lambda_{D}=0.1$, assuming $b_{W} / r_{0}=0.5$. These conditions correspond to $h \simeq 7.5$, i.e. a regime such that correlated collisions are present. As can be seen, the calculation converges after only a few iterations.

\section{COMPARISONS}

We have applied the collision operator formula Eq. (30) to calculations of hydrogen line shapes in ideal cases. The effective propagator has been estimated from Eq. (32) and the simplification presented in Appendix A [Eq. (A4)] has been used. Figure 2 presents a plot of the Lyman- $\alpha$ line $(n=2 \rightarrow 1)$ broadened due to ions at $r_{0} / \lambda_{D}=0.1$, assuming $b_{W} / r_{0}=0.2$ (a) and 0.5 (b), obtained using the unified theory (binary approximation) and compared to that obtained within the renormalized model. These cases correspond to $h \simeq 1.6$ and 7.5 , 


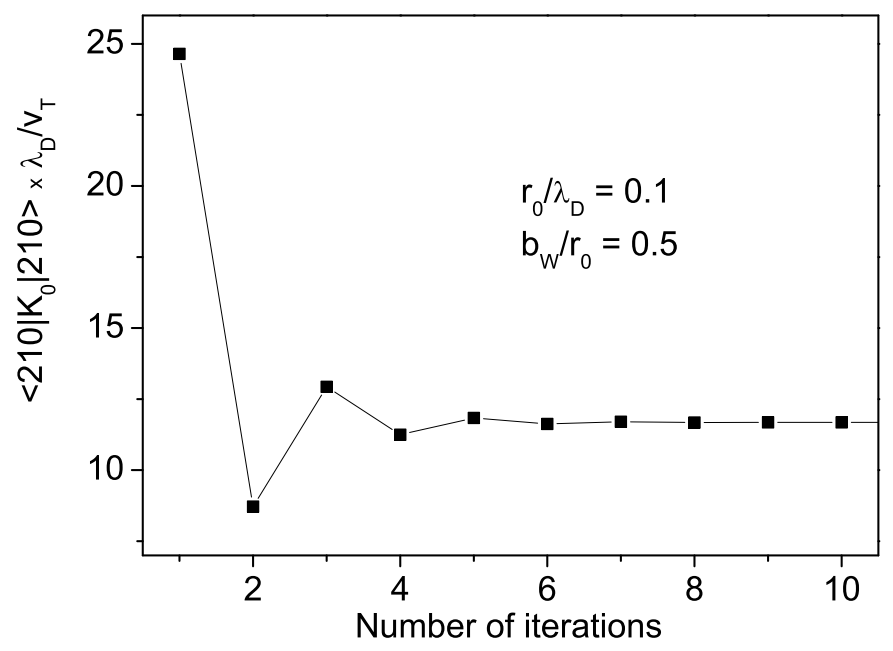

FIG. 1: Diagonal matrix element of $K_{0}$ for Ly- $\alpha$ in terms of the number of iterations for $r_{0} / \lambda_{D}=$ 0.1 , assuming $b_{W} / r_{0}=0.5$. The calculation converges after only a few iterations.

respectively. A numerical result from an ab initio simulation code is also shown in the figure. This code uses the "collision-time technique", which has been developed and designed in the past so as to provide correct statistics at regimes where $b_{W} / r_{0} \ll 1$ [34] (in particular, it avoids the bias induced by some prescribed boundary conditions). The technique assumes particles moving along straight lines, in agreement with the quasi-particle model used in the collision operator model. As can be seen, the binary model overestimates the width and predicts a different shape structure, with a dip at the center that increases with $b_{W} / r_{0}$. This dip is a consequence of the inadequacy of the binary model. Correlated collisions are important in this region because they govern the average atomic evolution operator at long times, hence, by virtue of the Laplace transform, at small frequency detuning. The typical range for the dip corresponds to frequencies smaller than the matrix elements of $K$ (see discussion in Appendix B). The renormalized model gives a much better result, with no dip and with an overestimate of the width no larger than $10 \%$ at $b_{W} / r_{0}=0.5$. Practically, this better result stems from the presence of $K$ into the denominator in Eq. (31). The small discrepancy remaining at the center is due to the part of the correlations that is not retained in the resummation procedure. This discrepancy increases with the ratio $b_{W} / r_{0}$. 

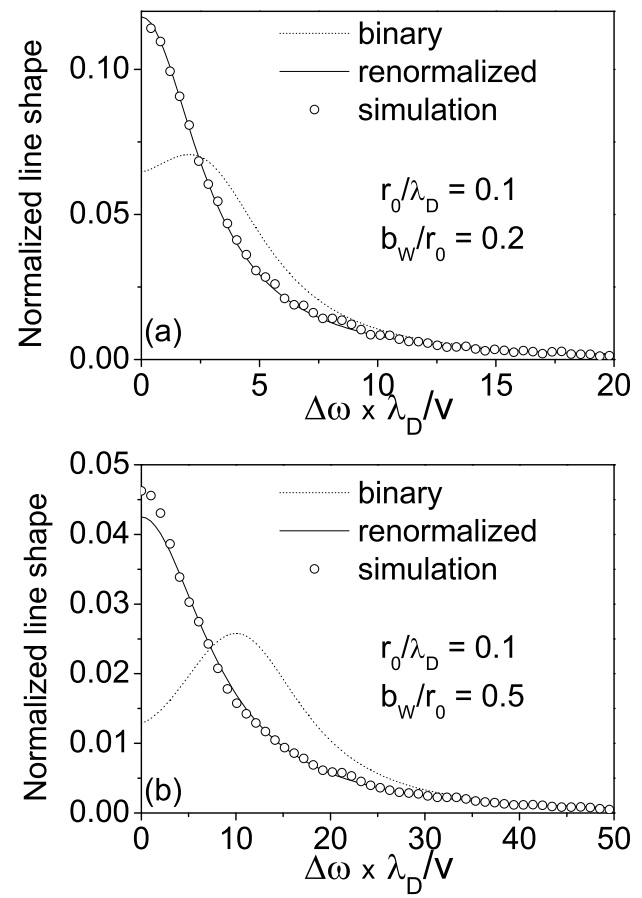

FIG. 2: Profile of Ly- $\alpha$ at $b_{W} / r_{0}=0.2$ (a) and 0.5 (b), i.e. when correlated collisions are present. The binary model overestimates the width and predicts a different shape, with a dip at the center that increases with $b_{W} / r_{0}$. The renormalized model gives a much better result, with no dip and with an overestimate of the width no larger than $10 \%$ at $b_{W} / r_{0}=0.5$.

\section{APPLICATION TO REALISTIC CASES}

We consider an application of the model to realistic cases in the framework of magnetic fusion research. Observations of spectral lines with a high upper principal quantum number (typically $n>7$, and up to $n=20$ in some cases) have been reported on in experiments involving recombining plasmas, in Alcator C-Mod [35-38], in ASDEX Upgrade [39], in NAGDIS-II [40-42], in JET [43], and in NSTX [44, 45]. For such lines, the ratio $b_{W} / r_{0}$ for the electrons can be as high as 0.5 and the $h$ parameter can be of the order of unity. This makes the binary approximation questionable. We have calculated the profile of the Ly ${ }_{20}$ transition $(n=20 \rightarrow 1)$ at $N_{e}=4 \times 10^{13} \mathrm{~cm}^{-3}$ and $T_{e}=T_{i}=0.2 \mathrm{eV}$ (typical conditions of divertor recombining plasmas), using the binary approximation and the renormalized model. The ionic contribution has been retained through statistical average over 
the microscopic electric field (quasi-static model). Practically, this amounts to making the formal substitution

$$
\begin{gathered}
L_{0} \rightarrow L_{0}-\mathbf{d} \cdot \mathbf{E}_{i} \equiv L_{0}-d_{z} E_{i}, \\
I(\omega) \rightarrow I\left(\omega, E_{i}\right),
\end{gathered}
$$

and to calculate the total line shape from the following integral

$$
I_{\mathrm{tot}}(\omega)=\int_{0}^{\infty} d E_{i} W\left(E_{i}\right) I\left(\omega, E_{i}\right),
$$

where $W\left(E_{i}\right)$ is the probability density function of the ionic electric field. For the latter, we have used the Hooper model [46], which is suitable for moderately coupled plasmas such as those of magnetic fusion experiments. The profile obtained within the binary approximation again differs from that obtained within the renormalized model. The dip present at the center stems from the neglect of correlated collisions. The typical frequency range is given by the matrix elements of $-i K-d_{z} E_{0}$ (with $E_{0}$ being the Holtsmark microfield). In a diagnostic context, this means that the use of the binary unified theory could lead to significant errors in the interpretation of spectra. We have also applied the model to the ion broadening of Ly- $\alpha$ in the atom's frame of reference (i.e. without Doppler broadening), motivated by opacity calculations for ITER modeling (e.g. [47, 48]). The plasma conditions expected in the ITER divertor are such that the ions are in a "near impact" regime, with $b_{W} / r_{0} \lesssim 0.5$ [49]. The typical magnetic field is so strong that the Zeeman effect splits the line into well separated components. Figure 4 shows a plot of the central component obtained with the two models at $N_{e}=2 \times 10^{15} \mathrm{~cm}^{-3}$ and $T_{e}=T_{i}=1 \mathrm{eV}$. Here again, the use of the binary approximation yields an incorrect result with a dip at the center. The influence of correlated collisions on Stark broadening is not specific to Lyman lines. We have applied the model to the $\mathrm{H}-\alpha$ transition $(n=3 \rightarrow 2)$, a line in the visible range. Figure 5 shows a plot of the spectral profile in the atom's frame of reference at $N_{e}=10^{15} \mathrm{~cm}^{-3}$ and $T_{e}=T_{i}=1 \mathrm{eV}$. Only the transition $3 d \rightarrow 2 p$ is presented in the figure, and no magnetic field is retained. The profile has been compared to numerical simulations. As can be seen, the binary theory strongly overestimates the width and again provides a dip. The renormalized model is in a much better agreement, with a discrepancy not larger than $10 \%$. 


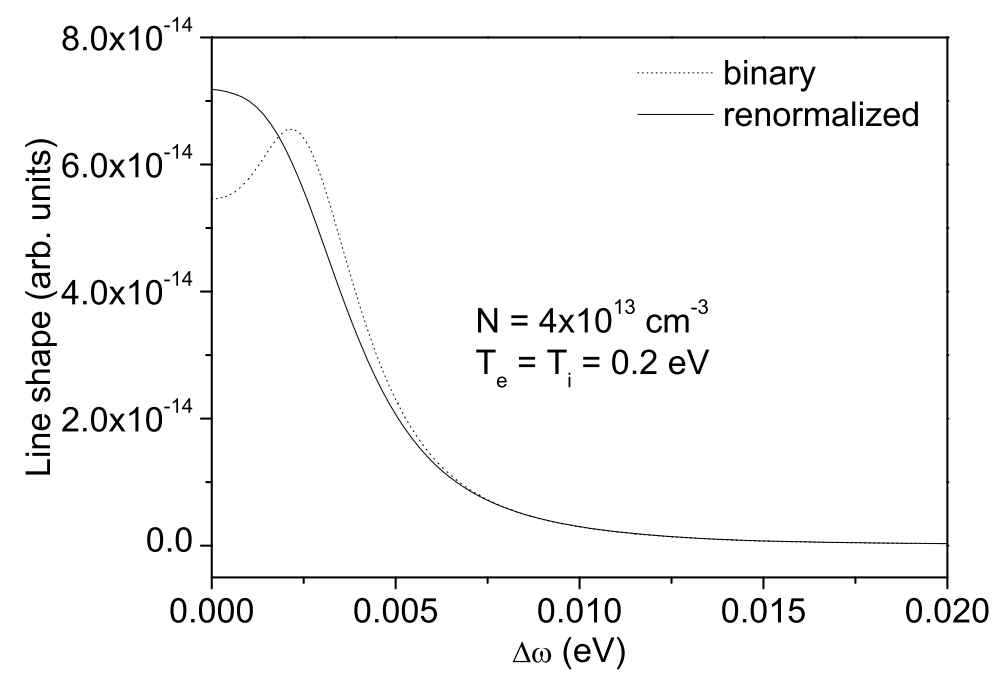

FIG. 3: Profile of $\mathrm{Ly}_{20}$ in recombining divertor plasma conditions. The dip present at the center stems from the neglect of correlated collisions. No dip is present if the renormalized model is used.

\section{CONCLUSION}

We have investigated the role of correlated collisions on Stark-broadened line shapes in plasmas using a kinetic-theory approach. Correlated collisions occur if the collision frequency becomes of the same order as or larger than the plasma frequency. In the model, the average atomic evolution operator is obtained from a hierarchy of equations similar to BBGKY. The presence of correlated collisions results in a renormalization of the atomic energy levels considered in a binary model. The results of the present model are in a very good agreement with numerical simulations. In contrast, the use of a binary model at conditions such that correlated collisions are present yields an erroneous line shape structure, with a dip present at the center. The method presented in this work provides an important correction to the usual models based on collision operators. The renormalization gives a structure of the new collision operator convenient for physical interpretation and suitable for numerical calculations, in particular for an implementation in line shape codes that use a binary collision operator (e.g. $[20,50])$. The model can be applied either to ions or electrons, and it can be generalized to isolated lines. Also, the kinetic theory treatment is transposable to quantum plasma [51]. 


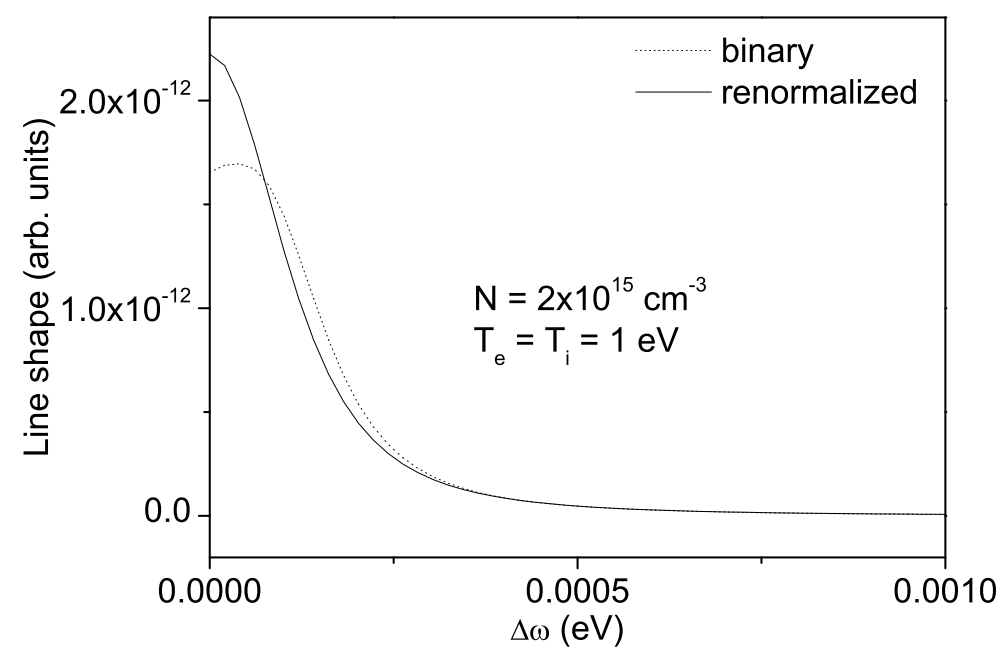

FIG. 4: Profile of Ly- $\alpha$, without Doppler broadening, in plasma conditions relevant to the ITER divertor. Only the central Zeeman component is shown here. The binary model again overestimates the width and predicts a different shape.

\section{Acknowledgments}

This work was carried out within the framework of the European Fusion Development Agreement and the French Research Federation for Fusion Studies. It is supported by the European Communities under the contract of Association between Euratom and CEA. The views and opinions expressed herein do not necessarily reflect those of the European Commission.

\section{APPENDIX A}

We derive here a simplification of the collision operator formula applicable to the hydrogen Lyman lines in the limit of small $r_{0} / \lambda_{D}$. We assume that collisions with a small impact parameter (strong collisions) give no significant contribution to the integrals in Eq. (30). On the other hand, we consider that the atomic wavefunction is not sensitive to perturbers located at large distance. In this framework, an approximation for the evolution operator is 


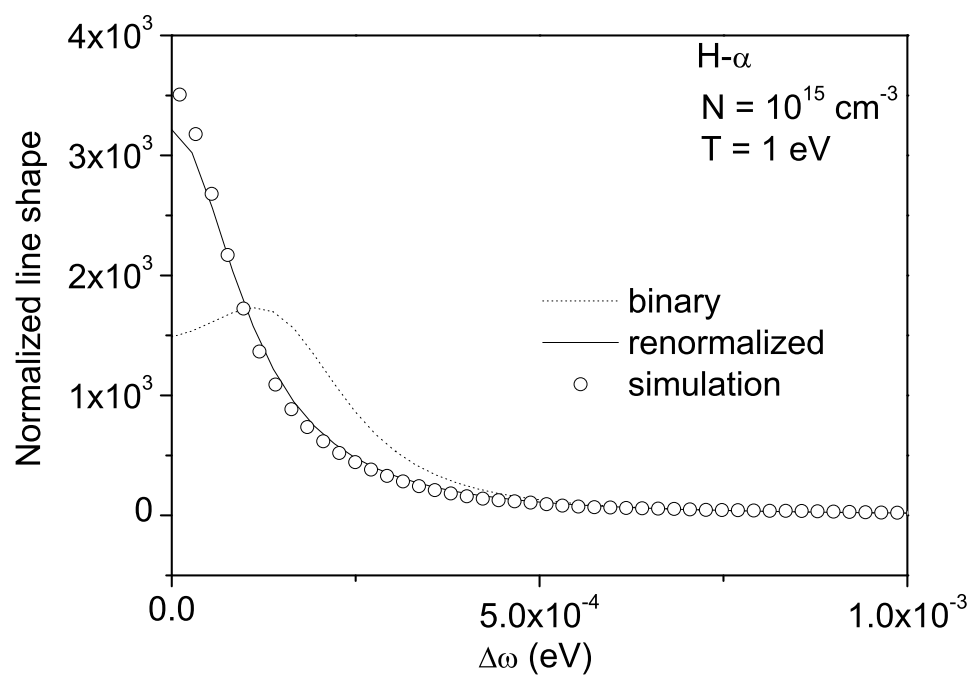

FIG. 5: The renormalized model can also be applied to Balmer lines. Here, plot of the 3d-2p transition with no Doppler broadening and no magnetic field.

provided by

$$
Q(1, t) \simeq\left\{\begin{array}{ll}
0 & \text { if } r_{1}<r_{m} \\
e^{-i L_{0} t} & \text { otherwise }
\end{array},\right.
$$

where $r_{m}$ is a characteristic radius that remains to be determined. A possible approach consists in estimating the matrix elements of the integral $\int_{0}^{t} d \tau e^{i L_{0} \tau} V\left(\mathbf{r}_{1}+\mathbf{v}_{1} \tau\right) e^{-i L_{0} \tau}$ that appears in the Dyson series and comparing them to unity [52]. Such a procedure yields the following relation

$$
r_{m}= \begin{cases}b_{W 1} & \text { if } b_{W 1}<v_{1} t \\ \sqrt{b_{W 1} v_{1} t} & \text { otherwise }\end{cases}
$$

where $b_{W 1}=\hbar n^{2} / m_{e} v_{1}$ is the Weisskopf radius specific to velocity $v_{1}$. This relation exhibits the separation between the short and long time regimes, which correspond to incomplete and complete collisions, respectively. The interaction term has been estimated as $V\left(\mathbf{r}_{1}+\mathbf{v}_{1} \tau\right) \sim$ $H\left(r_{m} / v_{1}-\tau\right) b_{W 1} v_{1} / r_{m}^{2}, H$ being the Heaviside function. From the approximation Eq. (A1), the space integral in Eq. (30) can be calculated analytically, in a fashion similar to what is done for the electric field autocorrelation function (e.g. [53]). We assume a Coulomb field and retain Debye screening through a cut-off at $\lambda_{D}$. Hence,

$$
\int d^{3} r_{1} V\left(\mathbf{r}_{1}+\mathbf{v}_{1} t\right) Q_{\mathrm{eff}}(1, t) V(1)
$$




$$
=\frac{4 \pi\left(b_{W 1} v_{1}\right)^{2}}{3\left(n^{2} e a_{0} / \hbar\right)^{2}} \mathbf{d} \cdot e^{\left(-i L_{0}-K_{0}\right) t} \mathbf{d} \times\left\{\begin{array}{ll}
\left(1 / r_{m}-1 / \lambda_{D}\right) & \text { if } v_{1} t \leq r_{m} \leq \lambda_{D} \\
\left(1 / v_{1} t-1 / \lambda_{D}\right) & \text { if } r_{m} \leq v_{1} t \leq \lambda_{D} \\
0 & \text { otherwise }
\end{array} .\right.
$$

The time integral in Eq. (30) can be evaluated analytically. The matrix element $\gamma(s)=$ $\langle n 10|K(s)| n 10\rangle$, which provides the line width and shift of Lyman lines, is given by

$$
\begin{aligned}
\gamma(s)= & \frac{4 \pi N\left(b_{W} v\right)^{2}}{3\left(n^{2} e a_{0}\right)^{2}} \sum_{l m}|\langle n 10|\mathbf{d}| n l m\rangle|^{2} \\
\times & \left\{\left[\operatorname{erf}\left(\frac{b_{W}}{\lambda_{D}}\right)-\frac{2}{\sqrt{\pi}} \frac{b_{W}}{\lambda_{D}} e^{-\left(b_{W} / \lambda_{D}\right)^{2}}\right]\left[\sqrt{\frac{\pi}{s^{\prime} b_{W} v}} \operatorname{erf}\left(\sqrt{\frac{\lambda_{D}^{2} s^{\prime}}{b_{W} v}}\right)-\frac{1-e^{-\lambda_{D}^{2} s^{\prime} / b_{W} v}}{\lambda_{D} s^{\prime}}\right]\right. \\
& +\int_{b_{W} v / \lambda_{D}}^{\infty} d v_{1} F\left(v_{1}\right)\left[\sqrt{\frac{\pi}{s^{\prime} b_{W} v}} \operatorname{erf}\left(\frac{\sqrt{s^{\prime} b_{W} v}}{v_{1}}\right)+\frac{1}{v_{1}} E_{1}\left(\frac{s^{\prime} b_{W} v}{v_{1}^{2}}\right)-\frac{1}{v_{1}} E_{1}\left(\frac{\lambda_{D} s^{\prime}}{v_{1}}\right)\right. \\
& \left.\left.-\frac{1-e^{-\lambda_{D} s^{\prime} / v_{1}}}{\lambda_{D} s^{\prime}}\right]\right\},
\end{aligned}
$$

where $b_{W}$ is the Weisskopf radius evaluated at the thermal velocity $v=\sqrt{2 k_{B} T / \mu}$ ( $\mu$ is the reduced mass of the emitter-perturber system), $s^{\prime}=-i \Delta \omega+\left\langle n l m\left|K_{0}\right| n l m\right\rangle$ with $\Delta \omega$ being the frequency detuning, $F\left(v_{1}\right)=4\left(v_{1} / v\right)^{2} \exp \left[-\left(v_{1} / v\right)^{2}\right] /(v \sqrt{\pi})$ is the Maxwellian velocity distribution function, and erf, $E_{1}$ are the error and first exponential integral functions, respectively. The Hilbert space formalism is used here, i.e. the bras, kets, and operators refer to atomic states instead of dyadics in the Liouville space. Equation (A4) is suitable for numerical calculations. The binary approximation ("usual" unified theory) corresponds to setting $s^{\prime} \equiv-i \Delta \omega$, and the complete collision approximation ("impact" theory) corresponds to setting $s^{\prime} \rightarrow 0$. An explicit calculation for $b_{W} \ll \lambda_{D}$ yields

$$
\gamma(s) \simeq \frac{4 \sqrt{\pi} N b_{W}^{2} v}{3\left(n^{2} e a_{0}\right)^{2}} \sum_{l m}|\langle n 10|\mathbf{d}| n l m\rangle|^{2}\left\{2+E_{1}\left[\left(\frac{b_{W}}{\lambda_{D}}\right)^{2}\right]\right\},
$$

which corresponds to the standard result for hydrogen [9]. In the case where a static electric field is present (Sec. V), the substitution Eq. (33) leads to the presence of a static Stark effect term in the Liouvillian in Eq. (A3). This term can be accounted for in the formula Eq. (A4) by using a decomposition onto the parabolic base. This procedure yields the following substitutions

$$
\begin{aligned}
& \sum_{l m} \ldots \rightarrow \sum_{l m n_{1}}\left|\left\langle n l m \mid n_{1} n_{2} m\right\rangle\right|^{2} \times \ldots \\
& s^{\prime} \rightarrow s^{\prime}-i\left\langle n_{1} n_{2} m\left|d_{z} E\right| n_{1} n_{2} m\right\rangle / \hbar
\end{aligned}
$$


where $n_{1}, n_{2}$ are the parabolic quantum numbers. A similar procedure can be applied to the other matrix elements of the collision operator, which are required if a static Stark effect term is present.

\section{APPENDIX B}

An analysis of the failure of the binary model when correlated collisions are present can be performed from an estimate using a simplification of the collision operator formula Eq. (A4). We ignore the velocity average, so that $F\left(v_{1}\right)$ can be replaced by $\delta\left(v_{1}-v\right)$, and we assume $b_{W}^{\prime} \ll \lambda_{D}$. We also ignore the imaginary part and we focus on frequencies much smaller than $v / b_{W}$ and larger than $v / \lambda_{D}$, in such a way to investigate a region dominated by incomplete weak collisions. With these orderings the diagonal matrix elements of the binary collision operator can be evaluated as follows

$$
\begin{gathered}
\gamma(\Delta \omega) \simeq \gamma_{0} \ln \left(\frac{v}{b_{W} \Delta \omega}\right), \\
\gamma_{0}=\frac{4 \pi N b_{W}^{2} v}{3\left(n^{2} e a_{0}\right)^{2}} \sum_{l m}|\langle n 10|\mathbf{d}| n l m\rangle|^{2} .
\end{gathered}
$$

This expression is similar to the Lewis model [23]. The line shape is given by

$$
I(\Delta \omega)=C \frac{\gamma(\Delta \omega)}{\pi} \frac{1}{\Delta \omega^{2}+\gamma(\Delta \omega)^{2}},
$$

where $C$ is a normalization constant. A dip occurs if the line shape has extrema at a finite value for $\Delta \omega$, larger than $v / \lambda_{D}$. The resolution of the equation $d I(\Delta \omega) / d \Delta \omega=0$ shows that this occurs for $\Delta \omega=\gamma(\Delta \omega) / \sqrt{\left(1+2 \ln \left(v / b_{W} \Delta \omega\right)\right)}$. The right-hand side of this expression can be estimated taking a constant value $\Lambda$ of the order of unity for the logarithm. This provides a position for the local maximum of the line shape and a criterion for the presence of a dip. If $\Lambda$ is estimated such that $\Lambda / \sqrt{(1+2 \Lambda)}=\ln \left(\lambda_{D} / b_{W}\right)$, this criterion reduces to $h \gtrsim 1$ where $h$ is the parameter introduced in the main text.

[1] R. Balescu, Statistical Dynamics - Matter Out of Equilibrium (Imperial College Press, London, 1997).

[2] G. G. Lister, J. E. Lawler, W. P. Lapatovich, and V. A. Godyak, Rev. Mod. Phys. 76, 541 (2004). 
[3] B. Omar, S. Günter, A. Wierling, and G. Röpke, Phys. Rev. E 73, 056405 (2006).

[4] E. Stambulchik, S. Alexiou, H. R. Griem, and P. C. Kepple, Phys. Rev. E 75, 016401 (2007).

[5] J. E. Bailey, G. A. Rochau, R. C. Mancini, C. A. Iglesias, J. J. MacFarlane, I. E. Golovkin, C. Blancard, P. Cosse, and G. Faussurier, Phys. Plasmas 16, 058101 (2009).

[6] D. Reiter, S. Wiesen, and M. Born, Plasma Phys. Control. Fusion 44, 1723 (2002).

[7] C. A. Iglesias, V. Sonnad, B. G. Wilson, and J. I. Castor, High Energy Density Phys. 5, 97 (2009).

[8] H. R. Griem, Principles of Plasma Spectroscopy (Cambridge University Press, Cambridge, 1997).

[9] H. R. Griem, A. C. Kolb., and K. Y. Shen, Phys. Rev. 116, 4 (1959).

[10] M. Baranger, Phys. Rev. 112, 855 (1958).

[11] W. L. Wiese, D. E. Kelleher, and V. Helbig, Phys. Rev. A 11, 1854 (1975).

[12] R. Stamm, Y. Botzanowski, V. P. Kaftandjian, B. Talin, and E. W. Smith, Phys. Rev. Lett. 52, 2217 (1984).

[13] M. A. Gigosos, J. Fraile, and F. Torres, Phys. Rev. A 31, 3509 (1985).

[14] S. Alexiou, Phys. Rev. Lett. 76, 1836 (1996).

[15] E. Stambulchik, High Energy Density Phys. 6, 9 (2010).

[16] A. Brissaud and U. Frisch, J. Quant. Spectrosc. Radiat. Transfer 11, 1767 (1971).

[17] C. Stehlé, Astron. Astrophys. Suppl. Ser. 104, 509 (1994).

[18] B. Talin, A. Calisti, L. Godbert, R. Stamm, R. W. Lee, and L. Klein, Phys. Rev. A 51, 1918 (1995).

[19] A. Calisti, C. Mossé, S. Ferri, B. Talin, F. Rosmej, L. A. Bureyeva, and V. S. Lisitsa, Phys. Rev. E 81, 016406 (2010).

[20] S. Ferri, A. Calisti, C. Mossé, L. Mouret, B. Talin, M. A. Gigosos, M. A. Gonzalez, and V. S. Lisitsa, Phys. Rev. E 84, 026407 (2011).

[21] D. Voslamber, Z. Naturforsch. 24a, 1458 (1969).

[22] E. W. Smith, J. Cooper, and C. R. Vidal, Phys. Rev. 185, 140 (1969).

[23] M. Lewis, Phys. Rev. 121, 501 (1961).

[24] J. W. Dufty, Phys. Rev. A 2, 534 (1970).

[25] S. Günter, L. Hitzschke, and G. Röpke, Phys. Rev. A 44, 6834 (1991).

[26] S. Ferri, A. Calisti, R. Stamm, B. Talin, R. W. Lee, and L. Klein, Phys. Rev. E 58, R6943 
(1998).

[27] H. Capes and D. Voslamber, Phys. Rev. A 5, 2528 (1972).

[28] H. Capes and D. Voslamber, Phys. Rev. A 15, 1751 (1977).

[29] J. Rosato, H. Capes, Y. Marandet, A. Mekkaoui, and R. Stamm, Transport Theor. Stat. Phys. 41, 337 (2012).

[30] R. Balescu, Equilibrium and Nonequilibrium Statistical Mechanics (Wiley-Interscience, New York, 1975).

[31] T. H. Dupree, Phys. Fluids 9, 1773 (1966).

[32] J. Weinstock, Phys. Fluids 12, 1045 (1969).

[33] N. D. Mermin, Phys. Rev. B 1, 2362 (1970).

[34] G. C. Hegerfeldt and V. Kesting, Phys. Rev. A 37, 1488 (1988).

[35] B. L. Welch, H. R. Griem, J. Terry, C. Kurz, B. LaBombard, B. Lipschultz, E. Marmar, and G. McCracken, Phys. Plasmas 2, 4246 (1995).

[36] D. Lumma, J. L. Terry, and B. Lipschultz, Phys. Plasmas 4, 2555 (1997).

[37] B. Lipschultz, J. L. Terry, C. Boswell, A. Hubbard, B. LaBombard, and D. A. Pappas, Phys. Rev. Lett. 81, 1007 (1998).

[38] A. Y. Pigarov, J. L. Terry, and B. Lipschultz, Plasmas Phys. Control. Fusion 40, 2055 (1998).

[39] U. Wenzel, K. Behringer, A. Carlson, J. Gafert, B. Napiontek, and A. Thoma, Nucl. Fusion 39, 873 (1999).

[40] N. Ohno, D. Nishijima, S. Takamura, Y. Uesugi, M. Motoyama, N. Hattori, H. Arakawa, N. Ezumi, S. Krasheninnikov, A. Pigarov, et al., Nucl. Fusion 41, 1055 (2001).

[41] D. Nishijima, U. Wenzel, K. Ohsumi, N. Ohno, Y. Uesugi, and S. Takamura, Plasma Phys. Control. Fusion 44, 597 (2002).

[42] S. Takamura, N. Ohno, D. Nishijima, and Y. Uesugi, Plasma Sources Sci. Technol. 11, A42 (2002).

[43] M. Koubiti, S. Loch, H. Capes, L. Godbert-Mouret, Y. Marandet, A. Meigs, R. Stamm, and H. Summers, J. Quant. Spectrosc. Radiat. Transfer 81, 265 (2003).

[44] V. A. Soukhanovskii, D. W. Johnson, R. Kaita, and A. L. Roquemore, Rev. Sci. Instrum. 77, 10F127 (2006).

[45] F. Scotti, V. A. Soukhanovskii, M. L. Adams, H. A. Scott, H. W. Kugel, R. Kaita, and A. L. Roquemore, J. Nucl. Mater. 415, S405 (2011). 
[46] C. F. Hooper, Phys. Rev. 165, 215 (1968).

[47] V. Kotov, D. Reiter, A. S. Kukushkin, H. D. Pacher, P. Börner, and S. Wiesen, Contrib. Plasma Phys. 46, 635 (2006).

[48] J. Rosato, D. Reiter, V. Kotov, Y. Marandet, H. Capes, L. Godbert-Mouret, M. Koubiti, and R. Stamm, Contrib. Plasma Phys. 50, 398 (2010).

[49] J. Rosato, Y. Marandet, H. Capes, S. Ferri, C. Mossé, L. Godbert-Mouret, M. Koubiti, and R. Stamm, Phys. Rev. E 79, 046408 (2009).

[50] M. L. Adams, R. W. Lee, H. A. Scott, H. K. Chung, and L. Klein, Phys. Rev. E 66, 066413 (2002).

[51] D. Voslamber, Phys. Lett. 40A, 266 (1972).

[52] R. Stamm and D. Voslamber, C. R. Acad. Sc. Paris Série B t. 280, 223 (1975).

[53] A. Brissaud, C. Goldbach, J. Léorat, A. Mazure, and G. Nollez, J. Phys. B: At. Mol. Phys. 9, $1129(1975)$. 